Article

\title{
The moderating effect of parenting on adaptation of children with leukemia
}

\author{
Marta Tremolada ${ }^{1,3}$, Livia Taverna ${ }^{2}$, Sabrina Bonichini ${ }^{1}$, Maria Caterina Putti ${ }^{3}$, Manuela Tumino ${ }^{3}$, Alessandra \\ Biffi $^{3}$ \\ 1 Department of Development and Social Psychology, University of Padua, Padua, Italy; marta.tremolada@unipd.it; \\ s.bonichini@unipd.it \\ 2 Faculty of Education, Free University of Bolzano-Bozen, Brixen, Italy; livia.taverna@unibz.it \\ ${ }^{3}$ Department of Child and Woman Health, University of Padua, Padua, Italy; mariacaterina.putti@unipd.it; \\ manuela.tumino@aopdveneto.it; alessandra.biffi@unipd.it \\ * Correspondence; marta.tremolada@unipd.it Tel. +393474868835
}

\begin{abstract}
Parents' attitudes and practices may support the children's reactions to treatments for leukemia and their general adjustment. This study has two aims: to explore parenting depending on child's age and to develop and test a model on how family processes influence the psycho-social development of children with leukemia.

Patients were 118 leukemic children and their parents recruited at the Haematology-Oncologic Clinic of the Department of Pediatrics, University of Padua. All parents were Caucasian with a mean age of 37.39 years $(\mathrm{SD}=6.03)$. Children's mean age was 5.89 years $(\mathrm{SD}=4.21)$.

After the signature of the informed consent, the parents were interviewed using the EFI-C from which we derived Parenting dimension and 3 parental perceptions on child's factors. One year later, the clinical psychologist interviewed again parents using the Vineland Adaptive Behavior Scales (VABS).

The analyses revealed the presence of a significant difference in parenting by child's age: Infants required a higher and more intensive parenting. Child's coping to medical procedures at the second week after the diagnosis, controlled for parenting effect, impacted upon child's adaptation one-year post diagnosis.

Specific intervention programmes are proposed in order to help children more at risk just after the diagnosis for developmental delays.
\end{abstract}

Keywords: children; leukaemia; in treatment; parenting; adaptation; moderation effect

\section{Introduction}

The main task of parenting is to give care. Caring for children involves responsibility for their well-being and knowledge about their needs and how to accommodate them. Most importantly, there is an emotional bond between parents and their children that is essential to children's development and happiness [1]. The central task for parents is not simply to keep the child alive, or provide appropriate discipline, but "create the conditions in which children can develop their fullest capacity both inside and outside the family" [2]. The responsibilities and challenges involved in childcare are numerous and parents usually find ways to manage it, even in extreme conditions, such when a child has cancer.

Having a child with cancer is an overwhelming life experience for both parents. It causes psychological distress, especially at the time of diagnosis but also throughout all the therapy cycles, that can impact on parental health locus of control [3] and can bring to post-traumatic stress symptoms [4-5].

The main task of parenting is to give care. Caring for children involves responsibility for their well-being and knowledge about their needs and how to accommodate them. The responsibilities and challenges involved in childcare are numerous and parents usually find ways to manage it, even in extreme conditions, such when a child has cancer. Taking care of the child with leukaemia is physically and emotionally demanding because parents must negotiate a myriad of potentially stressful situations daily: Talking with the child about the illness, learning how to correctly perform an home care regimen, managing the parent's own emotions about 
the child's disease and survival, advocating on the child's behalf within a complex health care system, providing care for unaffected siblings, and fulfilling extra-familiar obligations (employment, financial) during periods of child sickness or hospitalization [6]. 'Being there' is described as a parental response to the perceived vulnerability of the child and the parental need to give meaning to parenthood. It serves two purposes: protection and preservation. Protection means guarding the child against the negative aspects of illness and treatment. Preservation refers to the way parents influence the child's perception of his/her life, thus contributing to his/her coping and willingness to undergo treatment, to maximise the chances for survival [7].

Especially for mothers, caring evokes an intense emotional interdependence with their sick child, and involves a range of technical tasks and emotional work, including acting as "brokers" of information for their child and managing the cooperation with treatment [8]. When a child has cancer, parents enter a world where the terrain is unfamiliar and their basic childrearing tasks are challenged. Problems such as overprotectiveness, difficulty with consistent discipline and expression of appropriate concerns about "spoiling" the child, can occur in parenting tasks [9]. Parents of the pre-schooler children with Acute Lymphoblastic Leukemia (ALL) reported significantly more lax parenting practices and strategies associated with their child's sleep (i. co-sleeping, comforting activities, and offering food and drink in the bedroom than the healthy group) [10] that can bring also to children's sleeping difficulties. Also parents respond to child executive function difficulties with greater overprotection, which may be adaptive but not conducive to the development of independence. Although most parents report normative levels of child vulnerability and overprotection, a small subset demonstrate parenting practices that may place some survivors at risk for adverse outcomes [11].

Adherence to pediatric cancer treatment can be difficult for families, especially when the child is a young child and the required tasks include medical procedures (mouth care and physical exams) [12]. More important, some of these difficulties are related to the parent's childrearing attitudes and practices, with the supportive parenting style as the best one, encouraging children to express their needs, physical and emotional reactions to the treatment [12].

Little attention has been, as yet, paid to the caring efforts of parents and if it could be an important moderator in children's adaptation to the daily new life after a diagnosis of cancer. The present study seeks insight the parenting practices in caring of their child with leukaemia.

\subsection{Aims}

The first aim of this study was to understand the parenting behaviours, the coping strategies and adaptability of children with leukemia along parental narratives and their presence in our participants.

The second aim was to identify if parenting behaviours were different in relation to child's age. At this purpose, we expected that supportive parenting style could be an essential tool especially when the child is a pre-schooler and the required tasks include medical procedures (mouth care, conducting physical exams, venepunctures, bone marrow aspirations). Probably, a more necessary and intensive care was needed for infants and toddlers, than for older children.

The third aim was to understand if child coping strategies and child adaptability were related to parenting styles and were associated with child's age. In the literature (i.e., [13]) we have seen that parents' behavior may affect the child's reactions to treatment and general adjustment in several ways: Helping children during medical procedures $[\mathbf{1 4}, \mathbf{1 5}]$, increasing their general compliance with treatment $[\mathbf{1 2}, \mathbf{1 6}]$.

A priority was to develop and test explicit models of how family processes influence the psychological development of children with chronic health conditions. Studies have emphasized the importance of family communication and support [17] and of general family factors in exacerbating or attenuating the impact of the disease on the child [18].

Parental social support may buffer the association between parent and child distress, even if the quality and the timing of support are not clearly established. Another factor linked to distress in children is the quality of family environment $[19,20]$ with cohesive and expressive families more capable of ensuring the adjustment of each family member, and thereby buffering parent and child distress [21].

Basing upon the existing literature, we expect that family factors and disease characteristics would be causal independent variables which impact upon children's adaptive behaviors post one year of child's therapies. 
In particular, we think that a general dimension such as Parenting (comprehensive of all the parental strategies to help children to cope with the illness) assessed at the beginning of the child's hospitalizations and treatments, can be an important mediator that impacts upon child's early coping to medical procedures and upon child's behavioral adaptation one year post diagnosis.

\section{Materials and Methods}

\subsection{Procedure}

The project was approved by the University of Padua, Psychology faculty with the protocol n. 4039. The families were contacted by a clinical psychologist during the first hospitalization of their children, about one week after the diagnosis. Project aims were explained and informed consent was asked for. Informal contacts with the participants were kept up on a daily basis, to provide support and motivation for the project. The parents were interviewed in a separate room of the Clinic. The questionnaires were filled in the child's room, with the clinical psychologist's assistance, during a quiet period of the day. The participants were informed that they were free to drop out at any moment of the study. Each family was contacted again

at the several established time points: 1 month later, 6 months later and 12 months later, but here we will show only the first and the last assessment steps. The assessments were carried out at the Day Hospital or in the library of the Clinic. The psychologist remained constantly in touch with the child and the family for the duration of the study, with frequent telephone contacts and direct contacts during the DH check-ups. Before the assessments, the psychologist contacted by telephone the parent to agree about the meeting. The data collection phase of the study required several steps. The timing followed two main events: the child's coping and parenting assessment in the second week after the communication of the diagnosis (T1) adopting the EFI$\mathrm{C}$, and 12 months later (T4) adopting the Vineland Adaptive Behavior Scales (VABS). The first contact took place while the child was under the initial therapy in the Clinic and the next assessment was done when the child and the family came for day hospital visits.

\subsection{Participants}

Patients were 118 children with leukemia and their parents recruited at the Haematology- Oncologic Clinic of the Department of Child and Woman Health, University of Padua. All parents were Caucasians with a mean age of 37.39 years $(\mathrm{SD}=6.03$ ). Most parents had 13 years of school $(50.8 \%) ; 32.2 \%$ had 8 years; $5.9 \%$ had college education; $9.3 \%$ had degree or diploma and $1.7 \%$ had 5 years of school. Parents' incomes were average (52.7\%), high $(24.1 \%)$ and low $(23.2 \%)$ for Italian norms, but above poverty. The average of job hours/weekly were mostly around $35(28.4 \%)$ and $45(22 \%)$, even if the major part of parents was temporarily relieved of their work or they were housewives $(43.4 \%)$. The parents who participated were mostly mothers $(\mathrm{N}=101)$ and only a few were fathers $(\mathrm{N}=17)$ because the mothers were more proximal to the child during hospitalization while fathers stayed with other siblings or continued to work to maintain the family. In the preliminary analysis we controlled the possible differences between fathers and mothers. There were no significant differences in our variables so we decided to consider them all together. Children's mean age was 5.89 years $(\mathrm{SD}=4.21$, range $=$ 1 year-17 years). Mostly children had Acute Lymphoblastic Leukemia (ALL) ( $=98$ ), while 20 had Acute Myeloid Leukemia (AML). At the following time-points we had a loss of participants due to several reasons: 11 deceased, 4 changed health center, 5 relapsed or were in grave illness situation at the assessment moment and only 5 families dropped out from the study (3.9\%).

\subsection{Analyses plan}

\section{Analyses Plan}

Descriptive statistics were used to address the first question. To answer the second question on how child's ages affects parenting behavior at T1, an ANCOVA was performed with: Parental perception on child's dimensions of EFI-C (Coping and Adaptability) and demographic factors (parent's school years, parent's mean of job days/a week, parent's Life Stress Events) as covariates; Age of the Child (three levels: 0-3 ys, N=32; 4-6 ys, $\mathrm{N}=45$; more than 7 ys, $\mathrm{N}=35$ ) as fixed factor; Parenting as dependent variable.

To answer the second question on which family, child and disease factors just after the diagnosis communication were responsible for long-term adaptation of children with leukemia after 1 year of treatments, three regression analyses, using Multiple regression for both moderation and mediation effects, 
were conducted. Baron and Kenny [22] illustrated the three multiple regression analyses testing mediation effects: The significance of the path "predictor A (Parental perception on child's coping) on the mediator B (Parenting)" was examined in the first regression; the significance of the path "predictor A (Parental perception on child's coping) on the dependent variable C (VABS global score)" was examined in the second regression; finally, predictor and mediator used simultaneously as predictors of dependent variable were tested in the last equation.

Regression approach was also used here for testing moderation effects. The predictor and moderator main effects were entered simultaneously into the regression equation first, followed by the interaction of the predictor and moderator. After preliminary analyses of Pearson's bivariate correlations between the several variables tested, a model of mediation effects following the Baron and Kenny's rule (three linear regressions) was performed to evaluate the family factors and disease characteristics at T1 which impacted upon children's adaptive behaviors (Vineland Adaptive Behavior Scales global score) one year post child's therapies. Post hoc Sobel test was used to control the signification of mediation effects.

\subsection{Instruments}

The EFI-Cancer (EFI-C; [23-25]) is a parent interview which explores the daily routines of family life and the salient concerns regarding how that routine is organised. The interview is a mix of conversation, probing questions by the interviewer and pre-planned questions. Participants use their words and emphases. The interviews start with a question such

as: 'Would you guide me through your daily life? What is your and your child's routine?' The EFI interview form flows from our theoretical and epistemological approach, which starts with the observation that the daily family routines and actions constitute adaptation tasks, in which various people participate. Such tasks

are carried out, in practice, according to the family resources available and through specific scripts or sets of actions, which are meaningfully linked to the beliefs and values of the broader ecology and culture and which show the emotions and motivations held in them. When the adaptation tasks are overloaded by negative emotion, as often occurred in our case, the participants can sometimes find relief during the interview process $[25,26]$.

A total of 98 items were extracted from the parental narratives and 11 major dimensions were identified, each of them with good internal consistency, three dealing with child experience and eight with the parent one. One-quarter of the total 118 interviews were coded with a score ranging from 0 (low presence of variable) to 8 (high presence of variable) by two independent judges, showing good Spearman inter-rater reliability (rho=0.833; $\mathrm{p}=0.001)$.

Vineland Adaptive Behavior Scales (VABS) [27, 28]

The VABS are useful in assessing an individual's daily functioning throughout several domains of adaptive functioning (personal and social). In this study we used The Interview Edition, Expanded Form, with 540 items. This form is administered to a parent or caregiver in a semi-structured interview format and it yields a more comprehensive assessment of adaptive behaviour.

Adaptive behaviours investigated are: Communication, Daily Living Skills, Socialization and Motor abilities. The Communication domain is comprised of three sub-domains: Receptive, Expressive, and Written Language. The Daily Living Skills scale includes the Personal, Domestic, and Community sub-domains. The Socialization scale is comprised of the Interpersonal, Play and Leisure, and Coping Skills sub-domains. The Motor scale includes Gross and Fine motor abilities.

Studies confirming the reliability and validity of the VABS have solidified this measure as one of the most widely used assessments of adaptive behaviour. Using a checklist to assess adaptive behavior may limit the information an interviewer gathers about the various activities involved in an individual's behavior. A checklist also may allow the respondent to bias the outcome of the assessment because he or she might not fully understand the intent of certain items or might not know the criteria for scoring.

Consciously or unconsciously, the respondent might choose scores that do not reflect the individual's true behaviors. Reliability studies indicate that the professionally conducted and scored interviews have higher reliability and validity than checklists. Each item is rated "2" (behavior is usually or 
habitually performed), "1" (sometimes or partly performed), or " 0 " (never performed). In addition, there is a code ("N") for cases when the child has never had the opportunity to perform the activity and a code ("DK") to use when the caregiver does not know if the child performed the activity. In this study we used the raw scores of each domains and of the total adaptation score. Psychometric properties for Expanded Form in the Italian standardization version revealed a good reliability and validity and it is adopted also in this clinical population in a precedent study [29].

\section{SES Questionnaire}

Parental education and occupational status were measured. In particular, the following variables were considered: Number of years of school achievement, type and average hours of job, economical status, number of familiars and sons in the family.

\section{Results}

\subsection{Parenting behaviours, coping strategies and adaptability of children with leukemia along parental narratives}

The first aim was designed to extract the typology and the frequency of parenting behaviours, child's coping and adaptability from the parental narratives. Table 1 shows the EFI-C dimensions taken into consideration in this study and their descriptive frequencies. We can see that parenting dimension is the higher one, attesting at a medium-high level, while the parental perceptions of child coping and adaptability placed at a lowmedium level.

Table 1 - EFI-C child and parent dimensions and relative descriptive statistics considered in the present study.

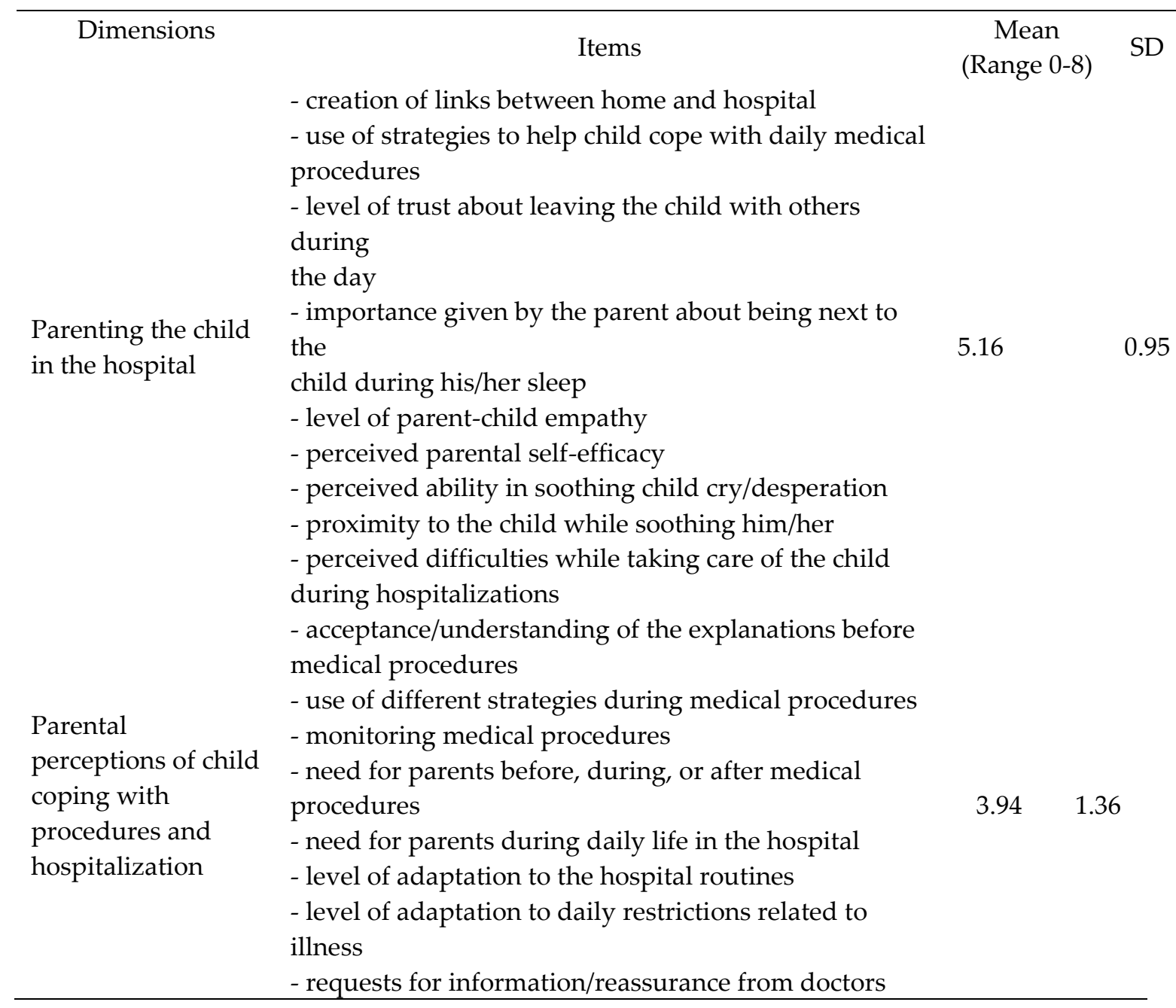


- coping with painful procedures

- tolerance to movement restrictions

- level of acceptance of the possible physical changes

- coping with emotional stress

- level of child's emotional intensity (crying, anger

episodes) associated with specific causes (medical

procedures)

- sleeping problems

- level of child's general curiosity and attention about

the hospital environment related with games and play

Parental perception of child

- level of child's consolable capacity

adaptability

- capacity of the child to become serene just after

medical interventions

- parent's perception about the quality of change in

child's relations with doctors and white coats

- parent's perception of a stability in child's

characteristics

- level of parent's perception of sane aspects of the child

in spite of the illness

The second question addressed from this study was: How child's ages affects parenting behavior at T1? To answer this question an ANCOVA was performed with: Parental perception on child's dimensions of EFI-C (Coping and Adaptability) and demographic factors (parent's school years, parent's mean of job days/a week, parent's Life Stress Events) as covariates; Age of the Child divided into three levels (0-3 ys; 4-6 ys; more than 7 ys) as fixed factor; Parenting as dependent variable. As expected, the analysis revealed the presence of a significant group difference on Parenting by Child's Age. When controlling for these independent variables, estimated marginal means for Parenting in the three groups of child's age were $5.56(\mathrm{SD}=0.14)$ for children aged 0-3, $5(\mathrm{SD}=0.09)$ for children aged 4-6 and $5.08(\mathrm{SD}=0.12)$ for the older children. Results are presented in Table 2 .

The third question was: Which family, child and disease factors just after the diagnosis communication are responsible for long-term adaptation of children with leukemia after 1 year of treatments? To answer this question three regression analyses, following Baron and Kenny (1986) rules, were used [20]. The significance of the path "predictor Child Coping on the mediator Parenting" was examined in the first regression $\left(\mathrm{R}^{2}=\right.$ $0.39 ; \beta=0.63 ; p=0.0001)$. The significance of the path "predictor Parental perception of child coping on the dependent variable Vineland Adaptive Behavior Scales (VABS) global score" was examined in the second regression $\left(R^{2}=0.39 ; \beta=0.62 ; p=0.0001\right)$; Finally, Parental perception of child coping $(\beta=0.69)$ and Parenting $(\beta=-0.11)$ were used simultaneously as predictors of VABS global score in the last equation $\left(R^{2}=0.40 ; p=\right.$ $0.0001)$. Mediation effect was measured by Sobel test $(Z=4.17 ; p=0.00003)$ (Figure 1$)$.

Table 2 ANCOVA comparing parenting between the three categories of child's age controlling for demographic factors and parental perception of child's dimensions

\begin{tabular}{|l|c|c|c|c|c|c|c|}
\hline \multicolumn{1}{|c|}{ Source } & $d f$ & $F$ & $\eta^{2} \boldsymbol{p}$ & $\boldsymbol{p}$ & $\boldsymbol{B}$ & $\boldsymbol{t}$ & Sig. \\
\hline Group (child's age): & 2 & 4.81 & 0.085 & 0.010 & 0.479 & 2.153 & 0.034 \\
in order 0-3; 4-6; 7 & & & & & & & \\
Parent schooling years & 1 & 0.62 & 0.006 & $\mathrm{~ns}$ & -0.076 & -0.492 & $\mathrm{~ns}$ \\
Parent mean of job days/a week & 1 & 0.07 & 0.001 & $\mathrm{~ns}$ & & & \\
Parent life stress events & 1 & 0.09 & 0.001 & $\mathrm{~ns}$ & & & \\
Parental perception of child's & 1 & 5.08 & 0.047 & 0.026 & & & \\
coping & & & & & & & \\
$\begin{array}{l}\text { Parental perception of child's } \\
\text { adaptability }\end{array}$ & 1 & 20.11 & 0.163 & 0.0001 & & & \\
\hline
\end{tabular}


Figure 1. Predictor and mediator factors tested at T1 of VABS global scale tested at T4.

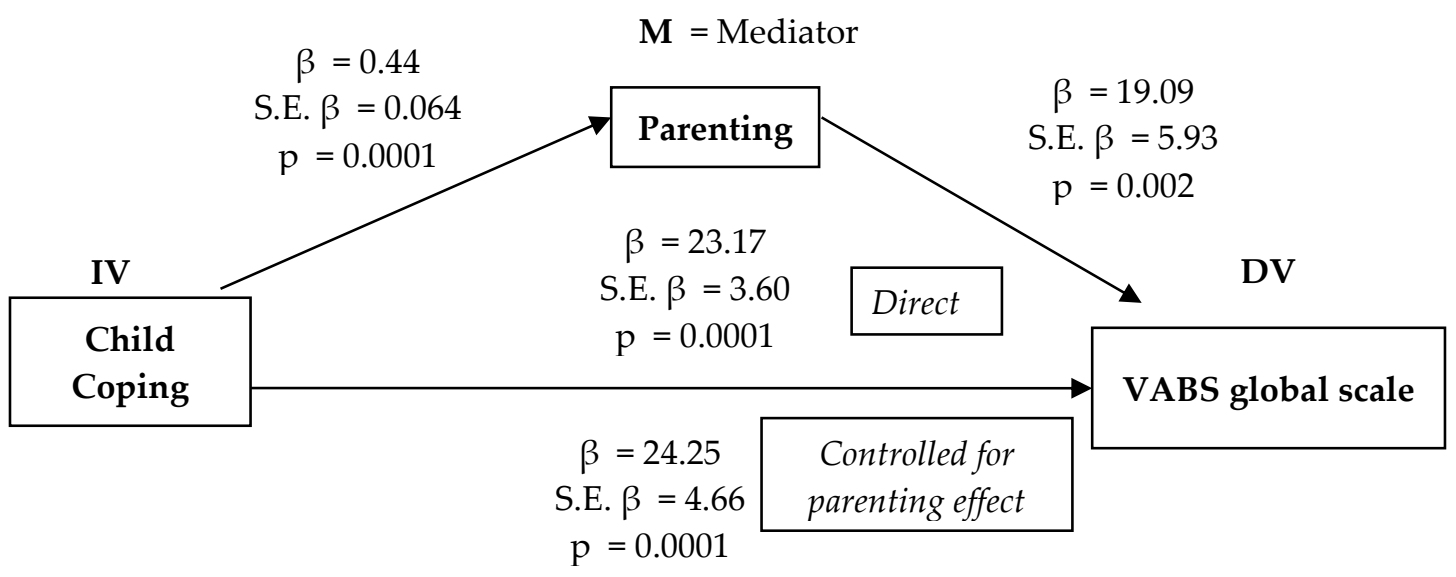

Legend: IV = Independent Variable; DV = Dependent Variable; $\mathrm{M}$ = Mediator.

Then, four hierarchical regression models were run to understand better which of the VABS domains were more significantly influenced by parental perception of child's coping and parenting. Two models obtained a significant prediction of both factors. The first had as dependent variable the Communication domain $\left(\mathrm{R}^{2}=\right.$ $0.42 ; p=0.0001)$ with Parental perception of child's coping $(\beta=0.79, p=0.0001)$ and parenting $(\beta=0.27$, $\mathrm{p}=0.008)$ impacting significantly on this. The second $\left(\mathrm{R}^{2}=0.28 ; \mathrm{p}=0.001\right)$ identified Parental perception of child's coping $(\beta=0.68, \mathrm{p}=0.0001)$ and parenting $(\beta=0.40, \mathrm{p}=0.001)$ as predictive factors of Motor skills.

\section{Discussion}

Parenting a child with cancer can be very difficult for families, especially when the child is very young and the required tasks include several medical and stressful procedures [10, 14]. As expected, the results of the present study confirm the presence of a significant group difference on parenting by child's age: Infants require a higher and more intensive parenting behavior, followed respectively by scholar-age children and by pre-scholar age children. We think that these findings confirm the difficult role of parents in caring their ill children when they are younger: Infants routines change so much, due to hospitalization in an important developmental time and they need more energy directed to them. Also the scholar age can be critical in another sense, because the children comprehend better the situation due to their cognitive maturity. This capacity sometimes is useful, but requires intensive parents' communication and support. Parents of school-aged children have to manage their parenting functions for helping their sons/daughters to tolerate the school and the peer relationship absences. Probably pre-scholar children have fewer needs in this sense, because they do not yet have many previous routines to maintain and they require primarily the physical presence of the parent into the hospital daily activities.

To answer the second research question about which family, child and disease factors just after the diagnosis communication are responsible for long-term adaptation of children with leukemia after 1 year of treatments, we examine the precedent studies, noting that in the literature there is no study that measures which family factors are responsible for long term child's behavioral adaptation at specific time points of the illness such as post 1 year. Our related priority is to develop and to test an explicit model of how family processes influence the psychological development of children with chronic disease conditions. So we expect that family factors and disease characteristics would be causal independent variables which impacts upon children's adaptive behaviors post one year of child's therapies. In particular, we think that parenting (comprehensive of all the parental strategies to help children to cope with the illness) assessed at the beginning of the child's hospitalizations and treatments, can be an important mediator that impacts upon child's early coping to medical procedures and upon child's behavioral adaptation one year post diagnosis. Our findings confirm the 
above hypotheses, showing that child's coping to medical procedures at the second week after the diagnosis controlled for parenting effect, impacts upon child's behavioral adaptation one year post diagnosis.

Parenting is a mediator that increases the effect of child's early coping upon child's adaptive functioning. Especially, parenting and parental perception on child's coping impact on Communication and Motor skills abilities. We can appreciate this result because a specific intervention programme can be implemented to help children more at risk just after the diagnosis for developmental delays. So these results prompt us to think about two parallel programmes: One to increase child's coping strategies during the first hospitalization, especially for school-aged children, and the other to teach some parenting strategies to parents of children just at the beginning of therapies, specific for the younger children.

Communication and motor abilities should be enforced adopting, just at the diagnosis time, the child's coping strategies (i.e. distraction, cognitive self-instruction, social support, problem solving). Parenting training could improve the emotional resources of parents for helping children in their adaptive tasks both at the beginning of the illness and after 1 year of therapies, when they could come back to their daily routines such as school or other social activities.

This study has several limits such the reduced number of participants, the unique centre experience, the adoption of proxy-report measures on pediatric patients without self-report ones. The strengths of this study are the homogeneity of the diagnosis of children (all leukemias), the richness of the qualitative data extracted from parental narratives, the longitudinal design of the study. The longitudinal timing is the authentic strength of the study because until now longitudinal studies about this topic are lacking; therefore, their research filled an important gap.

Recommendations for future research could include: involvement of other Haematology- Oncologic Clinics, the adoption also of self-report measures and standardized tests on the pediatric patients at the different time points and the assessment of efficacy of adoption of intervention programme on parenting or on child's coping.

Author Contributions: All authors have read and agree to the published version of the manuscript. Conceptualization, M.T. and S.B.; methodology, M.T. and S.B..; formal analysis, M.T.; investigation and data curation M.T., resources, S.B..; writing - original draft preparation, M.T.; writing - review and editing, L.T.; visualization, M.C.P. and M.T..; supervision, A.B.; project administration, S.B.

Funding: This research was funded by Istituto di Ricerca Città della Speranza, grant is actually expired.

Conflicts of Interest: The authors declare no conflict of interest. The funders had no role in the design of the study; in the collection, analyses, or interpretation of data; in the writing of the manuscript, or in the decision to publish the results.

\section{References}

(1) Ainsworth, M.D. (1969). Object relations, dependency, and attachment: a theoretical review of the infant-mother relationship. Child Development, 40, 969-1025.

(2) Cowan, P.A., Powell, D., and Cowan, C.P. (1997). Parenting interventions: A family systems perspective. In I. E. Sigel and K. A. Renninger (Eds.), Handbook of child psychology: Vol. 4. Child psychology in practice. (5th ed., pp. 3-72). New York: Wiley.

(3) Tremolada, M.; Taverna, L.; Bonichini, S.; Putti, M. C.; Pillon, M.; Biffi, A. Health Locus of Control in Parents of Children with Leukemia and Associations with Their Life Perceptions and Depression Symptomatology. Children (Basel) 2020, 7 (5). https://doi.org/10.3390/children7050040.

(4) Tremolada, M.; Bonichini, S.; Aloisio, D.; Schiavo, S.; Carli, M.; Pillon, M. Post-Traumatic Stress Symptoms among Mothers of Children with Leukemia Undergoing Treatment: A Longitudinal Study. Psychooncology 2013, 22 (6), 1266-1272. https://doi.org/10.1002/pon.3132.

(5) Tremolada, M.; Bonichini, S.; Schiavo, S.; Pillon, M. Post-Traumatic Stress Symptoms in Mothers of Children with Leukaemia Undergoing the First 12 Months of Therapy: Predictive Models. Psychol Health 2012, 27 (12), 1448-1462. https://doi.org/10.1080/08870446.2012.690414.

(6) Enskär, K.; Carlsson, M.; Golsäter, M.; Hamrin, E.; Kreuger, A. Life Situation and Problems as Reported by Children with Cancer and Their Parents. Journal of Pediatric Oncology Nursing 1997, 14 (1), 18-26. https://doi.org/10.1016/S1043-4542(97)90061-8.

(7) Kars, M. C.; Duijnstee, M. S. H.; Pool, A.; van Delden, J. J. M.; Grypdonck, M. H. F. Being There: 
Parenting the Child with Acute Lymphoblastic Leukaemia. J Clin Nurs 2008, 17 (12), 1553-1562. https://doi.org/10.1111/j.1365-2702.2007.02235.x.

(8) Young, B.; Dixon-Woods, M.; Findlay, M.; Heney, D. Parenting in a Crisis: Conceptualising Mothers of Children with Cancer. Soc Sci Med 2002, 55 (10), 1835-1847. https://doi.org/10.1016/s02779536(01)00318-5.

(7) Davies, W. H.; Noll, R. B.; DeStefano, L.; Bukowski, W. M.; Kulkarni, R. Differences in the ChildRearing Practices of Parents of Children with Cancer and Controls: The Perspectives of Parents and Professionals1. Journal of Pediatric Psychology 1991, 16 (3), 295-306. https://doi.org/10.1093/jpepsy/16.3.295.

(8) McCarthy, M. C.; Bastiani, J.; Williams, L. K. Are Parenting Behaviors Associated with Child Sleep Problems during Treatment for Acute Lymphoblastic Leukemia? Cancer Med 2016, 5 (7), 1473-1480. https://doi.org/10.1002/cam4.727.

(9) Sharkey, C. M.; Clawson, A. H.; Mullins, L. L.; Brinkman, T. M.; Pui, C.-H.; Hudson, M. M.; Krull, K. R. The Relationship of Child Executive Functions to Parenting Capacities in Childhood Acute Lymphoblastic Leukemia Survivors. Pediatr Blood Cancer 2019, 66 (8), e27761. https://doi.org/10.1002/pbc.27761.

(10) Manne, S. L.; Jacobsen, P. B.; Gorfinkle, K.; Gerstein, F.; Redd, W. H. Treatment Adherence Difficulties among Children with Cancer: The Role of Parenting Style. J Pediatr Psychol 1993, 18 (1), 47-62. https://doi.org/10.1093/jpepsy/18.1.47.

(11) Vance, Y.; Eiser, C. Caring for a Child with Cancer?A Systematic Review. Pediatric Blood E Cancer 2004, 42 (3), 249-253. https://doi.org/10.1002/pbc.10440.

(12) Jacobsen, P. B.; Manne, S. L.; Gorfinkle, K.; Schorr, O.; Rapkin, B.; Redd, W. H. Analysis of Child and Parent Behavior during Painful Medical Procedures. Health Psychol 1990, 9 (5), 559-576. https://doi.org/10.1037//0278-6133.9.5.559.

(13) Dahlquist, L. M.; Pendley, J. S.; Power, T. G.; Landthrip, D. S.; Jones, C. L.; Steuber, C. P. Adult Command Structure and Children's Distress during the Anticipatory Phase of Invasive Cancer Procedures. Children's Health Care 2001, 30 (2), 151-167. https://doi.org/10.1207/S15326888CHC3002_5.

(14) LaMontagne, L. L.; Wells, N.; Hepworth, J. T.; Johnson, B. D.; Manes, R. Parent Coping and Child Distress Behaviors during Invasive Procedures for Childhood Cancer. J Pediatr Oncol Nurs 1999, 16 (1), 3-12. https://doi.org/10.1177/104345429901600102.

(15) Rodrigue, J. R.; Behen, J. M.; Tumlin, T. Multidimensional Determinants of Psychological Adjustment to Cancer. Psycho-Oncology 1994, 3 (3), 205-214. https://doi.org/10.1002/pon.2960030307.

(16) Ostroff, J.; Ross, S.; Steinglass, P. Psychosocial Adaptation Following Treatment: A Family System Perspective on Childhood Cancer Survivorship. In In L. Baider, C.L. Cooper, A. Kaplan De-Nour (Eds), Cancer and the Family; John Wiley and Sons: Chichester, 2000; pp 155-173.

(17) Varni, J. W.; Katz, E. R.; Colegrove, R.; Dolgin, M. Family Functioning Predictors of Adjustment in Children with Newly Diagnosed Cancer: A Prospective Analysis. J Child Psychol Psychiatry 1996, 37 (3), 321-328. https://doi.org/10.1111/j.1469-7610.1996.tb01409.x.

(18) Drotar, D. Relating Parent and Family Functioning to the Psychological Adjustment of Children with Chronic Health Conditions: What Have We Learned? What Do We Need to Know? J Pediatr Psychol 1997, 22 (2), 149-165. https://doi.org/10.1093/jpepsy/22.2.149.

(19) Hammen, C.; Brennan, P. A.; Shih, J. H. Family Discord and Stress Predictors of Depression and Other Disorders in Adolescent Children of Depressed and Nondepressed Women. J Am Acad Child Adolesc Psychiatry 2004, 43 (8), 994-1002. https://doi.org/10.1097/01.chi.0000127588.57468.f6.

(20) Baron, R. M.; Kenny, D. A. The Moderator-Mediator Variable Distinction in Social Psychological Research: Conceptual, Strategic, and Statistical Considerations. J Pers Soc Psychol 1986, 51 (6), $1173-$ 1182. https://doi.org/10.1037//0022-3514.51.6.1173.

(21) Tremolada, M.; Axia, V.; Pillon, M.; Scrimin, S.; Capello, F.; Zanesco, L. Parental Narratives of Quality of Life in Children with Leukemia as Associated with the Placement of a Central Venous Catheter. J Pain Symptom Manage 2005, 30 (6), 544-552. https://doi.org/10.1016/j.jpainsymman.2005.05.021.

(22) Tremolada, M.; Bonichini, S.; Altoè, G.; Pillon, M.; Carli, M.; Weisner, T. S. Parental Perceptions of Health-Related Quality of Life in Children with Leukemia in the Second Week after the Diagnosis: A 
Quantitative Model. Support Care Cancer 2011, 19 (5), 591-598. https://doi.org/10.1007/s00520-0100854-5.

(23) Tremolada, M.; Bonichini, S.; Pillon, M.; Schiavo, S.; Carli, M. Eliciting Adaptive Emotion in Conversations with Parents of Children Receiving Therapy for Leukemia. J Psychosoc Oncol 2011, 29 (3), 327-346. https://doi.org/10.1080/07347332.2011.563341.

(24) Scrimin, S.; Axia, G.; Tremolada, M.; Pillon, M.; Capello, F.; Zanesco, L. Conversational Strategies with Parents of Newly Diagnosed Leukaemic Children: An Analysis of 4880 Conversational Turns. Support Care Cancer 2005, 13 (5), 287-294. https://doi.org/10.1007/s00520-004-0679-1.

(25) Sparrow, S.; Balla, D.; Cicchetti, D. Vineland Adaptive Behavior Scales Interview Edition: Survey Form Manual; American Guidance Service: Circle Pines, MN, 1984.

(26) Balboni, G.; Pedrabissi, L. Adattamento Italiano Delle Vineland Adaptive Behavior Scales.; Giunti Organizzazioni Speciali: Firenze, 2003.

(27) Tremolada, M.; Bonichini, S.; Basso, G.; Pillon, M. Development Delays and Temperament in Children with Leukemia after the First Year of Therapy. In In Alexandra M. Columbus (Ed.), Advances in Psychology Research.; Nova Science Publishers: New York, 2015; pp 55-70. 
